\title{
Extinction of conditioned electrodermal changes and human information processing'
}

VLADIMIR PISHKIN and JAY T. SHURLEY, VA Hospital and University of Oklahoma School of Medicine, Oklahoma City, Okla. 73104

An inhibitory effect of information load upon extinction of GSR of humans was reliably demonstrated. Antecedent failure on concept identification task reduced extinction rate of GSR as did complexity of a concomitant problem, in terms of bits of irrelevant information. Theoretical formulations regarding interaction of autonomic and cognitive functions in humans, in terms of Spence's inhibitory set and cybernetic aspects of the brain, were postulated.

In a series of experiments, Spence (1966) attempted to explain the differences in extinction rates of conditioned eye-blink in animal and human Ss. He pointed out succinctly that: "Indeed, it is strongly suspected that complex processes, call them cognitive factors, sets, mediating processes, or what you will, have greatly affected the extinction data heretofore reported for human subjects [Spence, 1966, p. 447]." Spence postulated the inhibitory set by humans as a function of their recognition of the shift from $100 \%$ to 0 reinforcement and suggested that introduction of a masking task, such as the probability learning task (Estes \& Straughan, 1954) should reduce the effects of the hypothetical inhibitory set during extinction. Accordingly, Spence, Homzie, \& Rutledge (1964) designed an experimental situation where discriminability of change in conditions from acquisition to extinction phases was minimized. They were successful in demonstrating that use of the masking task inhibited the influence of mediating sets resulting from S's recognition of the true purpose of the eye-blink extinction studies, i.e., by embedding the conditioning procedure within a learning task (Estes \& Straughan, 1954), they demonstrated that CR extinguished more slowly for Ss run within the masking paradigm than for those Ss exposed to standard conditioning extinction procedure.

Although the nature of the task in the present study differs from that of Spence's (1966), an examination of GSR conditioning and extinction phases in humans was attempted under conditions where GSR extinction was introduced concurrently with different levels of concept identifica- tion (CI) problem difficulty. Moreover, solvable and unsolvable sets were introduced during conditioning phases in order to examine the influence of cognitive sets upon GSR conditionability and extinction. A general hypothesis was generated on the basis of Spence's formulations (1966), related to eye-blink data, that GSR extinction rate would be reduced under conditions where Ss are involved in an information processing task. More specifically, set and complexity were manipulated within a CI task, concurrent with GSR extinction phase.

In an earlier study, Pishkin (1965) demonstrated that antecedent success and failure sets markedly influenced subsequent CI performance and found a significant interaction between set and task complexity. Accordingly, it is a plausible expectation that masking or inhibiting effects of CI task upon GSR extinction would vary with changes of set and complexity of information. One should expect reduced rates of extinction as a function of concomitant increases in information load (CI complex. ity) and of set, where S's universe of hypotheses for solution is greater. (Thus he is unable to isolate relevant information in an unsolvable set task.)

\section{METHOD}

The Ss were 30 male psychiatric patients with no history of psychotropic drug ingestion for 1 week prior to the experimental nun; they were between 20 and 55 years of age and were cooperative, had no history of brain damage, understood instructions, and were in general good contact. The conditioning and extinction procedures were identical to those described in detail (Pishkin, Shurley, \& Wolfgang, 1967) and consisted of two phases. In Phase 1 Ss were conditioned to a 2-sec, 500 -cps tone immediately followed by electric shock (subjectively determined pain level) for $2 \mathrm{sec}$ at 30 -sec intervals for a 10-min period concomitant with solvable or unsolvable set problem. In solvable set conditions, Ss were given correct feedback on every trial, while, in the unsolvable set, $50 \%$ misinformation feedback was provided.

In Phase $2 \mathrm{Ss}$ performed on a solvable CI task for $30 \mathrm{~min}$ and were concomitantly given 30 extinction trials (2-sec tone without shock at 1 -min intervals). There were three complexity conditions, with one, three, or five irrelevant dimensions, as described in an earlier study (Pishkin, 1965).
This was a 2 (set) by 3 (complexity) factorial design with five Ss randomly assigned to each of the six conditions.

RESULTS

The data were analyzed for CI errors in Phase 2, and for GSRs (both specific and spontaneous) in terms of conductance changes (Edelberg, 1967, pp. 1-53). Since there were no significant differences in either of the GSR measures of conditionability in Phase 1 , the data were analyzed for CI errors and GSR activity in Phase 2 only. Accordingly, the dependent variables analyzed were: (a) CI errors during Phase 2, (b) spontaneous GSRs (only GSRs equal to or greater than .001 of basal resistance were counted as spontaneous responses), and (c) specific GSRs (greatest conductance change within the 4-sec period beginning with the onset of a 2 -sec tone and the following 2-sec period without shock).

Analysis of variance on $\mathrm{CI}$ errors indicated that both main effects of complexity $[F(2,24)=15.62, p<.001]$ and set $[F(1,24)=9.83, p<.01]$ were significant. Moreover, Complexity by Set interaction was significant $[F(2,24)=10.81, p<.001]$, indicating that, as number of irrelevant dimensions increased, solution was progressively more difficult with the unsolvable, as compared to the solvable, set. The analysis of variance of spontaneous GSR data (all changes occurring in between 4-sec extinction trials) also showed significant main effects of complexity $[F(2,24)=5.36, p<.05]$ and set $[F(1,24)=6.42, p<.05]$. It is also noteworthy that, as in the previous study (Pishkin \& Shurley, 1968), the number of spontaneous GSRs significantly decreases with increase in complexity and that, as the number of irrelevant dimensions increases from one to five, the spontaneous GSRs are less frequent in the unsolvable set than in the solvable set conditions, as reflected by the Sex by Complexity interaction $[F(2,24)=6.31, p<.05]$.

As indicated in the introduction, the major purpose of this study was to examine GSR extinction rates under three complexity conditions and two sets which were introduced during conditioning phase. Figure 1 represents extinction curves under different conditions of complexity and set. The main effect of blocks of trials was significant $[F(4,120)=5.62, p<.001]$, indicating that overall (i.e., complexities and sets pooled) extinction occurred, Also, it is evident that there was differential extinction for the various levels of complexity as shown by a significant Complexity by Trials interaction $[\mathrm{F}(8,120)=3.42, \mathrm{p}<.001]$. Although Complexity by Set interaction was not significant, Trials by Set by Complexity interaction was $[F(8,120)=3.09, p<.01]$, documenting 
differential extinction rates over blocks of trials as a function of complexity and set.

The analysis of variance (specific GSRs) reveals that complexity $[F(2,24)=16.83$, $\mathrm{p}<.001]$ and set $[\mathrm{F}(1,24)=8.91, \mathrm{p}<.01]$ effects were significant, although there was no interaction between the two main effects. The response curves, as per Fig. 1, clearly demonstrate that the slopes of the extinction curves in the three complexity conditions differ markedly. It is evident that extinction rates are more rapid under less complex problems and that, when unsolvable set precedes extinction phase, the extinction rates are slower when compared to those in solvable set conditions.

\section{DISCUSSION}

Although in a previous study (Pishkin, Shurley, \& Wolfgang, 1967) set and complexity did not influence extinction of the specific GSRs, present findings clearly reveal significant complexity and set effects. In the unsolvable set condition of the earlier study (Pishkin, Shurley, \& Wolfgang, 1967) complexity was the same as in the Phase 2 , i.e., one, three, or five irrelevant dimensions; in the present study complexity of the set phase was always five irrelevant dimensions. Thus, high complexity (five dimensions) and unsolvable set interacted sufficiently to produce marked differentiation in extinction phases between the experimental conditions. It is also possible that the high complexity conditions (five irrelevant dimensions) during extinction were more similar to the original conditioning phase, which also had five irrelevant dimensions, resulting in slower extinction through a tendency for generalization from condition. ing to extinction.

In brief, the findings demonstrate increasing inhibition of extinction of conditioned GSR as a function of increasing complexity of concept identification problems, as well as a function of the antecedent set conditions. These data further support the view, derived from cybernetic theory of brain function, that all of the brain's "computing" capacity is used for current purposes. The essential psychobiological task of clearing out, here, in the waking state, no-longer-needed functional programs in the brain, Spence's (1966) theoretical "inhibitory set" or the extinction of a GSR appears to be delayed or inhibited when the structure of the set and of the cognitive task "masks" or does not permit the braincomputer to strike a quantitative probability balance in detection of changes in response-stimulus contingencies. Furthermore, it is noteworthy that the results show a reliable relationship between information load, i.e., number of irrelevant dimensions in concept identification, and extinction rates of GSR. It is clear that Spence's (1966) theorizing related to influence of cognitive

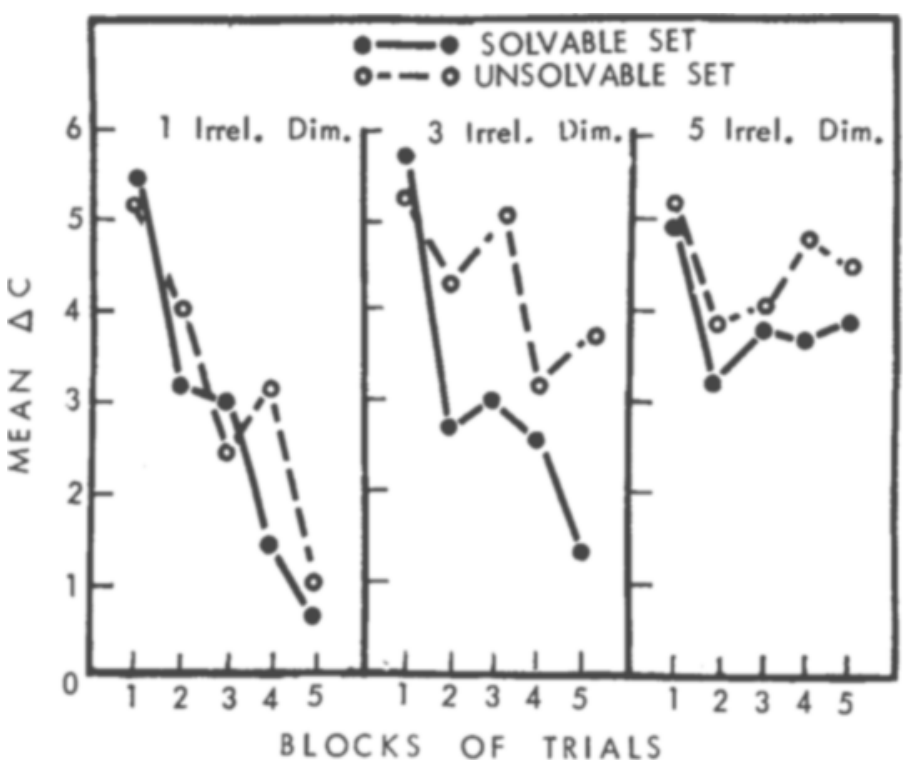

Fig. 1. Mean conductance changes in micromhos as functions of one, three, or five bits of irrelevant information and solvable or unsolvable sets. The data are represented in five trial blocks, each consisting of six ex tinction trials, over a 30-min period.

factors and sets on extinction data of conditioned eye-blink is supported strongly by the present findings with extinction of conditioned GSR responses.

\section{REFERENCES}

EDELBERG, R. Electrical properties of the skin. In C. C. Brown (Ed.), Methods in psychophysiology. Baltimore: The Williams \& Wilkins Co., 1967. Pp. 1-53.

ESTES, W. K., \& STRAUGHAN, J. W. Analysis of a verbal conditioning situation in terms of statistical learning theory. Joumal of Experimental Psychology, 1954, 47, 225-234.

PISHKIN, V. Dimension availability with antecedent success or failure in concept identification. Psychonomic Science, 1965, 2, 69-70.
PISHKIN, V., \& SHURLEY, J. T. Electrodermal and electromyographic parameters in concept identification. Psychophysiology, 1968, 5, $112-118$.

PISHKIN, V., SHURLEY, J. T., \& WOLFGANG, A. Stress. Archives of General Psy chiatry, 1967, $16,471-477$

SPENCE, K. W. Cognitive and drive factors in the extinction of the conditioned eye blink in human subjects. Psychological Review, 1966, 73, 445-458.

SPENCE, K. W., HOMZIE, M. J., \& RUTLEDGE, E. F. Extinction of the human eyelid $C R$ as a function of the discriminability of the change from acquisition to extinction. Journal of Experimental Psychology, 1964, 67, 545-552. NOTE

1. The authors are indebted to Robert Edelberg for his valuable suggestions.

\section{Decrease in polarity of semantic differential ratings as a function of previous trials with similar materials}

WILLIAM E. GUMENIK, The University of
Toledo, Toledo, Ohio 43606

Eighty eight Ss rated 10 good words and 88 Ss, 10 bad words on a 7-point good-bad semantic differential scale. In both cases, the polarity of the ratings decreased as a direct linear function of the number of words previously rated.
Osgood (1957, pp. 84-85) assumed that semantic differential ratings of an item are independent of the meaning of other items on the test form and thereby unaffected by anchor and central tendency effects. He reported an unpublished study by Aiken which supported this assumption. Investigators, on the basis of this assumption of independence, have often, as a matter of convenience, presented the items to be judged in a set order, even in the case of 